\section{Case Reports in Oncology}

\title{
Regressed Seminoma with Metastases to the Jejunum and Retroperitoneum
}

\author{
Nozomi Kashu Shoji Oura Naoki Kataoka Shinichiro Makimoto \\ Department of Surgery, Kishiwada Tokushukai Hospital, Kishiwada, Japan
}

\section{Keywords}

Jejunal metastasis $\cdot$ Regressed germ cell tumor $\cdot$ Regressed seminoma $\cdot$ Tumor-infiltrating lymphocytes

\begin{abstract}
A 66-year-old man with vomiting and weight loss was referred to our hospital. Abdominal computed tomography showed small bowel obstruction caused by a presumed small intestinal tumor. Single-balloon endoscopy showed an ulcerated tumor and marked stenosis of the jejunum. Immunohistochemical staining suggested the tumor to be poorly differentiated or undifferentiated carcinoma. The patient underwent open surgical resection of the jejunal tumor and regional lymph nodes both to improve the quality of life of the patient and to possibly get a cure of the presumed jejunal carcinoma. Pathological examination of the excised tumor and lymph nodes including para-aortic lymph nodes showed large-sized tumor cells and massive lymphocyte infiltrates. Immunostaining showed the tumor cells to be OCT3/4, AE1/AE3, CD117, and D2-40 positive, leading to the diagnosis of metastatic seminoma. With the preoperative diagnosis of a presumed burned-out tumor of the testis, the patient underwent left high orchiectomy. Pathological examination of the left testis showed marked scar tissue, no teratoma elements, and no residual tumor cells. Under the final diagnosis of regressed seminoma, the patient has received combination chemotherapy using bleomycin, etoposide, and cisplatin as adjuvant chemotherapy. Surgical oncologists should take regressed seminoma into their differential diagnosis when the biopsy specimens of the presumed intestinal malignancy show poorly differentiated or undifferentiated atypical cells with massive lymphocyte infiltrates, especially in postpubertal men. Confirmation of a malignant noninvasive component should be another important clue to the appropriate differential diagnosis when choosing between metastatic seminoma and poorly differentiated or undifferentiated intestinal primary malignancies.




\section{Introduction}

Seminoma is the most common testicular germ cell tumor, and many early - that is, stage 1 - testicular seminomas can be successfully treated with orchiectomy without adjuvant chemotherapy, called "active surveillance" [1]. Due to its high radio- and chemosensitivity, more advanced seminomas also show a favorable prognosis with surgery followed by some kind of adjuvant therapy [2]. In addition, even recurrent seminomas show much better survival outcomes, often presumed cures, with multidisciplinary therapy when compared with the outcomes of other solid malignancies such as gastric, colon, hepatic, pancreatic, and lung cancer.

It is well known that less than 5\% [3] - but a certain proportion - of testicular germ cell tumors regress spontaneously (regressed germ cell tumors). Regressed germ cell tumors cannot be detected when lacking any symptomatic local and/or distant lesion(s), strongly suggesting that the regressed germ cell tumors thus far diagnosed are just the tip of the iceberg.

We report here a case of regressed seminoma with metastases to the jejunum and its regional nodes.

\section{Case Report}

A 66-year-old man complaining of vomiting and weight loss was referred to our hospital. Abdominal positron emission tomography/computed tomography showed small bowel obstruction caused by a presumed small intestinal tumor, lymph node dissemination in the mesentery and para-aortic loci, and no increased avidity in other organs (Fig. 1a, b). Labo-

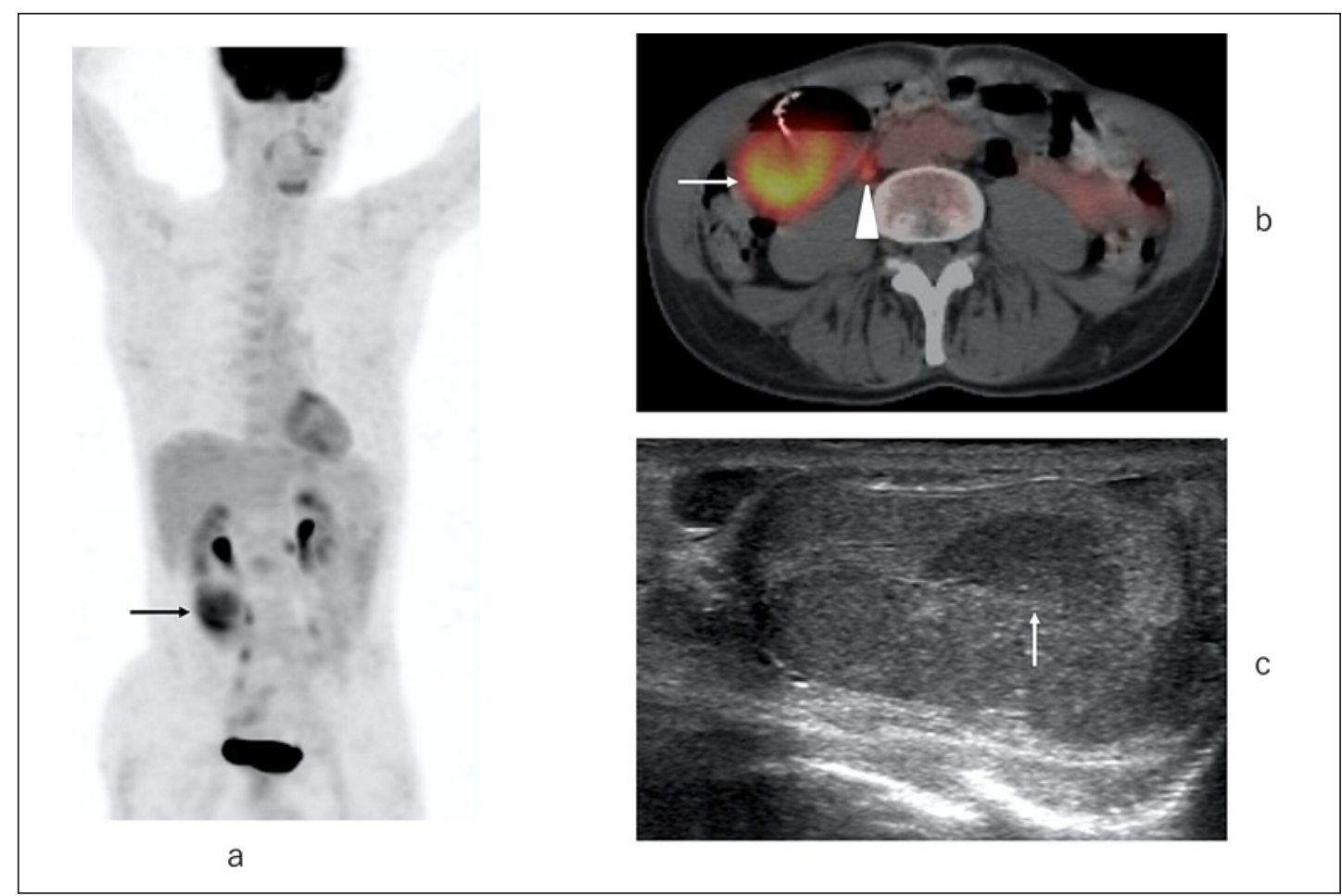

Fig. 1. Systemic and local imaging findings. a Coronal view of positron emission tomography (PET) showed an uptake (arrow) just caudal to the right kidney. b Axial view of PET/computed tomography showed jejunal dilatation and uptake both in the jejunum (arrow) and in the lymph node (arrowhead). c A hypointense area (arrow) was observed in the left testis on ultrasound. 


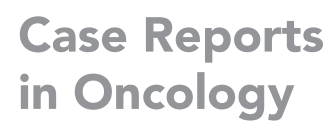

Kashu et al.: Regressed Seminoma with Metastases to the Jejunum and Retroperitoneum

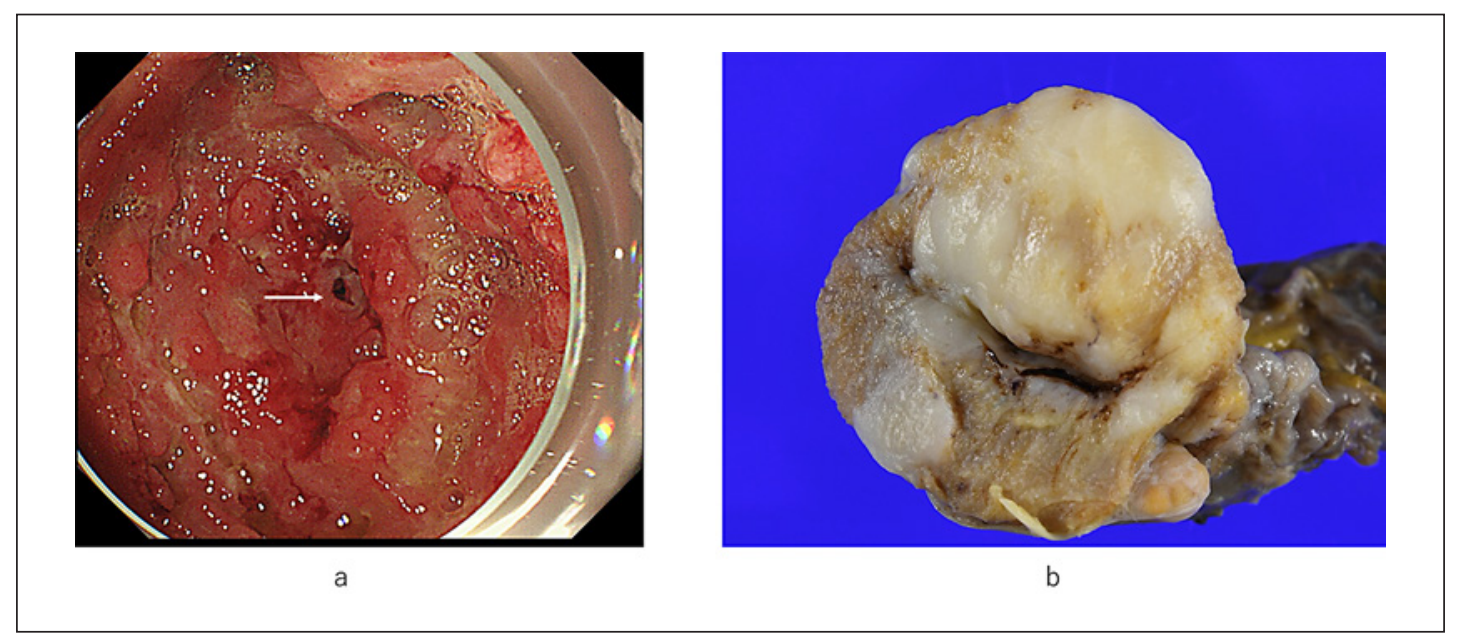

Fig. 2. Local findings in the jejunum and gross appearance of the left testis. a Marked stenosis (arrow) was observed in the massive ulcerated tumors on single-balloon endoscopy. b A yellowish-white nodule $25 \mathrm{~mm}$ in size was observed in the left testis.

ratory testing showed no abnormality in carcinoembryonic antigen, CA19-9, lactate dehydrogenase (LDH), and serum interleukin-2 levels. Single-balloon endoscopy (Fig. 2) showed an ulcerated tumor and marked stenosis of the jejunum (i.e., it was impossible to insert an endoscope), leading to placement of an ileus tube into the small bowel.

Pathological examination of the endoscopic biopsy specimen showed medium- to largesized oval atypical cells with pale-to-clear abundant cytoplasm, crisp cytoplasmic membranes, and one or more centrally located nucleoli with no epithelial atypia in the jejunal gland. Immunohistochemical staining showed the atypical cells to be LCA, melan A, HMB-45, CD56, synaptophysin, and chromogranin negative as well as AE1/AE3 positive, suggesting the tumor to be poorly differentiated or undifferentiated carcinoma. The patient, therefore, underwent open surgical resection of the jejunal tumor with lymph node dissection both to improve the quality of life of the patient and to possibly get a cure of the presumed jejunal carcinoma.

Pathological examination of the excised tumor showed large-sized tumor cells, with clear and abundant cytoplasm and large nucleoli interrupted by fibrous septa with massive lymphocyte infiltrates, proliferating in a sheet-like or trabecular fashion but lacking atypical noninvasive components (Fig. 3a, b). Immunostaining showed the tumor cells to be OCT3/4, AE1/AE3, CD117, and D2-40 positive, CK7 focally positive, and CD30 negative, leading to the diagnosis of metastatic seminoma. Magnetic resonance imaging of the left testis showed partially lower intensity and higher signals on the T2-weighted images and on the diffusionweighted images, respectively, than did imaging of the right testis. Ultrasonography showed a focal low area in the left testis (Fig. 1c) compared to the uniformly high-level internal echoes in the right testis. With the preoperative diagnosis of a presumed burned-out tumor of the testis, the patient underwent left high orchiectomy. Pathological examination of the left testis showed marked scar tissue, no teratoma elements, and no residual tumor cells including germ cell neoplasia in situ (Fig. 3c, d). Under the final diagnosis of regressed seminoma with jejunal and retroperitoneal metastases, the patient has received combination chemotherapy using bleomycin, etoposide, and cisplatin as adjuvant chemotherapy.

\section{Karger'}




\section{Case Reports in Oncology}

\begin{tabular}{l|l}
\hline Case Rep Oncol 2021;14:418-423 \\
\hline DOI: 10.1159/000513027 & $\begin{array}{l}\text { ○ 2021 The Author(s). Published by S. Karger AG, Basel } \\
\text { www.karger.com/cro }\end{array}$ \\
\hline
\end{tabular}

Kashu et al.: Regressed Seminoma with Metastases to the Jejunum and Retroperitoneum

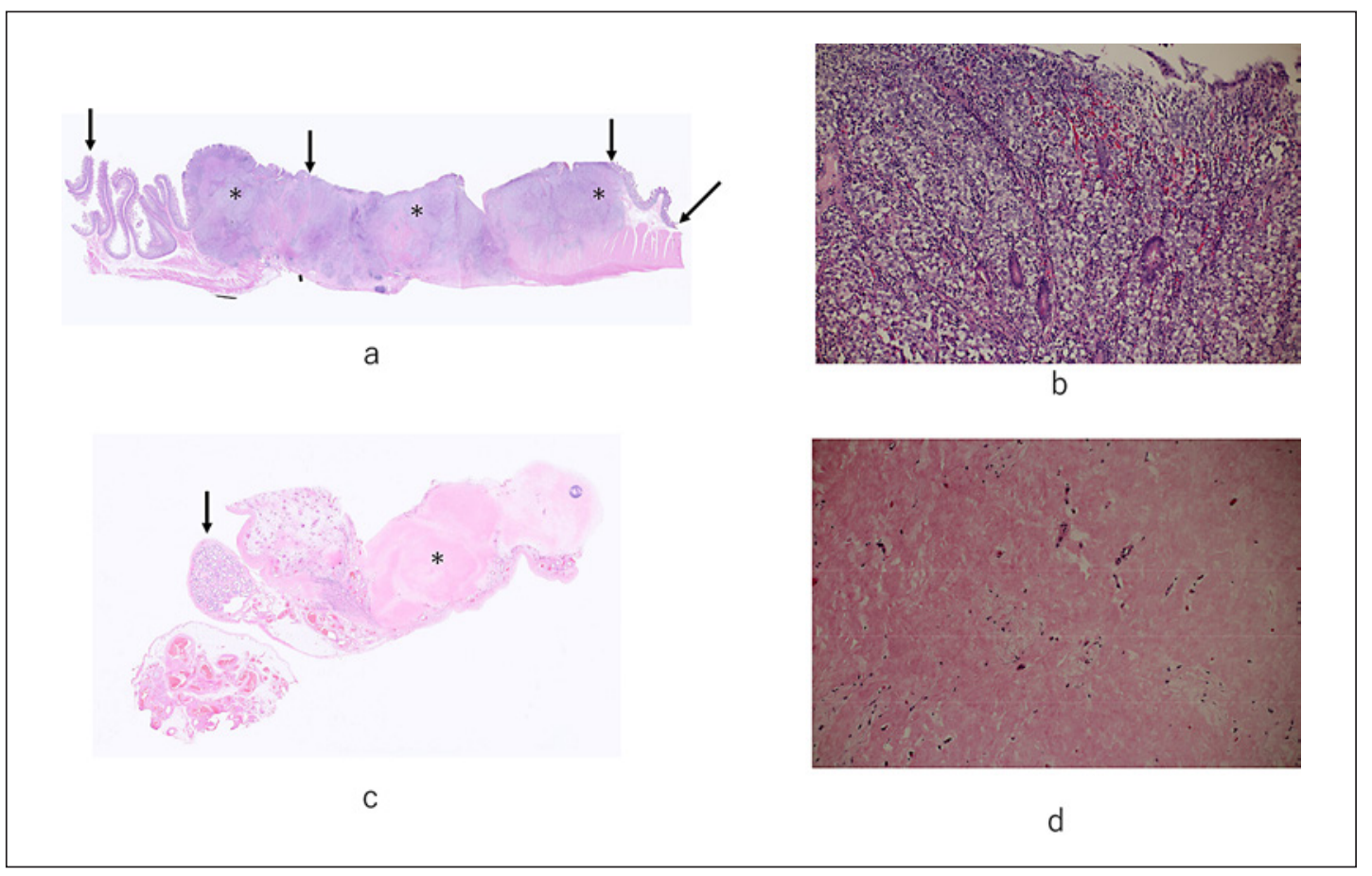

Fig. 3. Pathological examination. a Low-magnification view showing the jejunal tumor (asterisks) located in the jejunal submucosa with no noninvasive component. The jejunal epithelium (arrows) showed no atypia. b High-magnification view of the jejunal tumor showing large-sized tumor cells with a clear and abundant cytoplasm and large nucleoli proliferating in a sheet-like or trabecular fashion. Collagen fibers with massive lymphocytes intervened in the sheets and trabeculas. c Low-magnification view of the left testis showing no malignant cells, Leydig cells (arrow), and sclerotic scarring (asterisk). d High-magnification view of the left testis showing extremely hypocellular collagen fibers.

\section{Discussion}

Breast cancer, especially the human epidermal growth factor receptor type 2 (HER2)expressing subtype, strongly responds to anti-HER2 agent-containing chemotherapy. Approximately half the HER2-positive breast cancers generally show a pathological complete response to anti-HER2 agent-containing chemotherapy. Therefore, the nomenclature of primary systemic therapy [4] is used in the field of breast cancer oncology, instead of neoadjuvant chemotherapy, which is often used in the treatment of other solid malignancies. HER2type breast cancers, however, show quite different pathological complete response rates among those with and those without massive tumor-infiltrating lymphocytes in the tumor [5]. In this case, massive lymphocyte infiltration was observed in the jejunal metastatic foci.

Merkel cell carcinoma (MCC) is a highly aggressive cutaneous neuroendocrine carcinoma, often metastasizing to distant organs. In some extremely rare cases, however, patients develop spontaneous regression of the MCC just after incisional biopsy of the tumor [6]. It has already been proven that $\mathrm{T}$-cell-mediated immunity mainly contributes to the spontaneous regression of MCC [7]. Spontaneous regression of the seminoma probably contributed to the high prevalence of lymphocytes just around the tumor cells also seen in this case. Pathological examination of the left testis, however, showed no lymphocyte infiltrates in the presumed preexisting tumor locus. Seminoma often develops in patients with a mean age of around 40 years, and rarely in those older than 70 years [8]. Judged by the patient's age of 66 years, the presumed 
long interval from complete seminoma regression in the testis to onset of jejunal obstruction probably well explains the lack of lymphocyte infiltrates in the regressed seminoma.

We do not know what the long-term clinical outcome of this patient will be. In addition, we did not check serum alphafetoprotein and human chorionic gonadotropin levels, due to the lack of a preoperative differential diagnosis of regressed seminoma. Serum LDH levels, however, have been within the normal limits throughout the clinical course. It is well known that serum LDH is highly (i.e., approx. 80\%) elevated in seminoma patients, and a normal preoperative LDH level well suggests a favorable clinical outcome [9]. In addition, the presence of massive lymphocyte infiltrates in the jejunal lesion highly predicts the amazing efficacy - probably similar to that seen in tumor-infiltrating lymphocyte-rich HER2-positive breast cancers [5] - of adjuvant chemotherapy against possible microscopic seminoma cell remnants. We, therefore, will follow the patient after the completion of adjuvant chemotherapy with promising expectations.

The biopsy specimen suggested presumed undifferentiated or poorly differentiated malignant cells but no epithelial atypia in the jejunal gland. Seminoma favors the spread via lymphatics, initially to the retroperitoneum, followed by mediastinal and cervical, especially left supraclavicular, lymph nodes [10]. Regressed seminoma with distant metastases to the alimentary tract itself easily misleads the attending physician to diagnose the lesion(s) as primary malignancy. On the one hand, the presence of noninvasive malignant cells clearly implies primary malignancy, but on the other hand the absence of those in the tiny biopsy specimen does not negate primary malignancy. Physicians, however, should cautiously check the malignant in situ component to make a more accurate preoperative histological diagnosis of intestinal malignancies in addition to various immunostainings.

In conclusion, regressed seminoma is a very rare entity and needs careful diagnostic and therapeutic strategies, especially in postpubertal men. When present in men, massive lymphocyte infiltrates in the biopsy specimen with undifferentiated or poorly differentiated malignant cells suggest the need for a differential diagnosis of regressed germ cell tumor.

\section{Statement of Ethics}

We have reported this case in compliance with the Declaration of Helsinki. Written informed consent was obtained from the patient for the publication of this case report and any accompanying images.

\section{Conflict of Interest Statement}

The authors have no conflicts of interest to declare.

\section{Funding Sources}

No funding was received.

\section{Author Contributions}

N.K. contributed to the design of the report and collected the data. S.O. drafted the manuscript. N.K. decided how to treat the patient. S.M. revised the manuscript. All authors have read and approved the final version of the manuscript.

\section{Karger' ${ }^{\prime \prime}$}




\section{Case Reports in Oncology}

\begin{tabular}{l|l}
\hline Case Rep Oncol 2021;14:418-423 \\
\hline DOI: 10.1159/000513027 & $\begin{array}{l}\text { ○ 2021 The Author(s). Published by S. Karger AG, Basel } \\
\text { www.karger.com/cro }\end{array}$ \\
\hline
\end{tabular}

Kashu et al.: Regressed Seminoma with Metastases to the Jejunum and Retroperitoneum

\section{References}

1 Martin J, Chung P, Warde P. Treatment options, prognostic factors and selection of treatment in stage I seminoma. Onkologie. 2006;29(12):592-8.

2 Gholam D, Fizazi K, Terrier-Lacombe MJ, Jan P, Culine S, Theodore C. Advanced seminoma - treatment results and prognostic factors for survival after first-line, cisplatin-based chemotherapy and for patients with recurrent disease: a single-institution experience in 145 patients. Cancer. 2003;98(4):745-52.

3 Angulo JC, González J, Rodríguez N, Hernández E, Núñez C, Rodríguez-Barbero JM, et al. Clinicopathological study of regressed testicular tumors (apparent extragonadal germ cell neoplasms). J Urol. 2009;182(5):230310.

4 Sinclair S, Swain SM. Primary systemic chemotherapy for inflammatory breast cancer. Cancer. 2010;116(11 Suppl 1):2821-8.

5 Denkert C, von Minckwitz G, Brase JC, Sinn BV, Gade S, Kronenwett R, et al. Tumor-infiltrating lymphocytes and response to neoadjuvant chemotherapy with or without carboplatin in human epidermal growth factor receptor 2-positive and triple-negative primary breast cancers. J Clin Oncol. 2015;33(9):983-91.

6 Pang C, Sharma D, Sankar T. Spontaneous regression of Merkel cell carcinoma: a case report and review of the literature. Int J Surg Case Rep. 2015;7C:104-8.

7 Mori Y, Hashimoto K, Tanaka K, Cui CY, Mehregan DR, Stiff MA. A study of apoptosis in Merkel cell carcinoma: an immunohistochemical, ultrastructural, DNA ladder and TUNEL labeling study. Am J Dermatopathol. 2001; 23(1):16-23.

8 Berney DM, Warren AY, Verma M, Kudahetti S, Robson JM, Williams MW, et al. Malignant germ cell tumours in the elderly: a histopathological review of 50 cases in men aged 60 years or over. Mod Pathol. 200821;21(1): 54-9.

9 Fosså A, Fosså SD. Serum lactate dehydrogenase and human choriogonadotrophin in seminoma. Br J Urol. 1989;63(4):408-15.

10 Bredael JJ, Vugrin D, Whitmore WF Jr. Autopsy findings in 154 patients with germ cell tumors of the testis. Cancer. 1982;50(3):548-51. 